\title{
Rotating-Mirror Q-Switched Er:YAG Laser for Optodynamic Studies
}

\author{
Aleš Bizjak1, ${ }^{1, *}$ - Karolj Nemeš² - Janez Možina ${ }^{3}$ \\ 1 I.H.S. d.o.o., Slovenia \\ 2 Fotona d.d., Slovenia \\ 3 University of Ljubljana, Faculty of Mechanical Engineering, Slovenia
}

In this article a development of a simple, reliable and repeatable Q-switched Er:YAG laser with a pulse duration of $270 \mathrm{~ns}$ and energies up to $16 \mathrm{~mJ}$ in a TEM ${ }_{00}$ spatial beam mode is presented. The $Q$-switching is performed mechanically with a flat, highly reflective silicon rotating mirror. The laser pumping chamber is cooled with thermally stabilized de-ionized water. This laser is intended for optodynamic studies of the short Er:YAG laser pulses with various media, primarily those containing water, where the thermal diffusion effects are negligible.

(C)2011 Journal of Mechanical Engineering. All rights reserved.

Keywords: Er:YAG laser, Q-switch, inverse population, optodynamics, Xe flash lamp, rotating mirror

\section{INTRODUCTION}

The Er:YAG laser is a well-accepted tool in many medical applications, like the ablation of hard and soft dental tissue as well as in various areas of dermatology [1] to [3]. For all these applications the Er:YAG laser operates in a freerunning mode with laser-pulse durations from several tens of $\mu$ s up to several ms. The interaction is based on the coincidence of the Er:YAG laser wavelength $(2940 \mathrm{~nm})$ with the absorption peak of water (absorption coefficient $\mu_{a}=13,300$ $\mathrm{cm}^{-1}$ [4] and [5]. This high absorption leads to a very shallow (several $\mu \mathrm{m}$ ) penetration depth in the water as well as in all water-rich biological tissue. During the interaction of the laser pulses with the biological tissue, part of the laser-pulse energy is used up for ablation (depending on the instantaneous laser pulse power and the fluence), while the remaining part of the pulse energy during the laser pulse leaves the interaction region with diffusion that has a characteristic diffusion length of $(4 D T)^{1 / 2} \quad(D$ is the diffusivity of the media and $T$ is the laser-pulse duration). Recently, interactions in which the diffusion of heat from the interaction region during the laser pulse can be neglected became of some interest. Any study of this type of interaction obviously needs a veryshort-pulse Er:YAG laser.
Approaches for the reliable, Q-switched operation of an Er:YAG laser have already been described in the literature [6] to [8]. Most of these approaches need special materials and expensive components like undoped $\mathrm{Nd}$ :YAG crystals cut and polished at precise angles or specially designed electro-optic Q-Switches based on $\mathrm{LiNbO}_{3}$ crystals and high-voltage Q-switch drivers. The repeatability and long lifetime, as well as the easy set-up of the experimental laboratory, are important issues. Since the rotating-mirror and Q-switched lasers (Nd:YAG, Er:glass, and others) are already known for their reliability and repeatability, we decided to use this approach for the Q-switched Er:YAG laser as well.

The Er:YAG laser is a complex, rareearth, solid-state crystal laser in which $50 \%$ of the yttrium ions are replaced with $\mathrm{Er}^{3+}$ ions. The laser is exited with Xe flash lamps [9]. Since the laser operates at fairly high pumping energies, efficient cooling of both, the laser rod and the flash lamp is accomplished with de-ionized water. The Er:YAG laser is one of the rare lasers that operate at a wavelength near $3 \mu \mathrm{m}$ at room temperature.

The basic Q-switching technique for the rotating-mirror laser is well known and described elsewhere [10], and only the main principles are shown in Fig. 6. One of the resonator mirrors rotates at a constant frequency $\omega$ and passes the resonant alignment (with low losses) of the 
resonator once in a single rotation. The laser rod is excited with a Xe flash map until a steady inversion population $n$ is built up during the nonresonant condition of the laser. When the rotating mirror reaches the resonant position at time $t_{0}$, the resonator losses abruptly fall to a level where laser oscillation starts and a fast build-up of photons from the inverse population creates a Q-switched laser pulse (Fig. 1 photon flux $\Phi$ ).

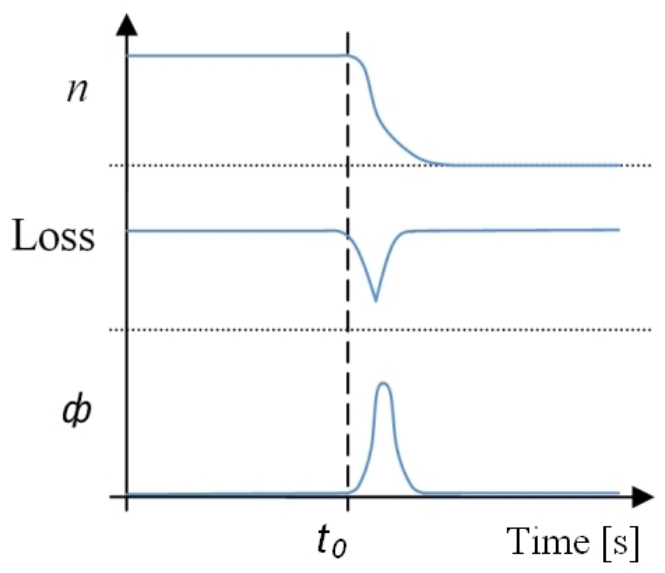

Fig. 1. Dynamics of the inverse population $n$ and the photon flux $\phi$, according to the resonator losses following the time $t_{0}$

In an analysis of the Q-switched laser it is very important to understand the laser crystal population dynamics, Q-switch operation itself as well as the laser resonator structure.

The Er:YAG crystal's laser energy levels are shown in Fig. 2.

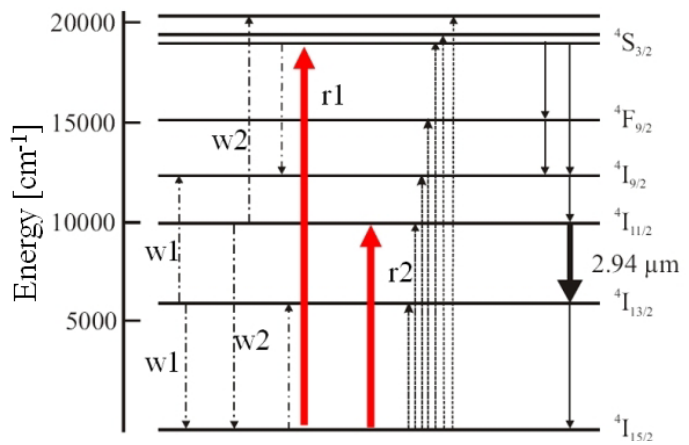

Fig. 2. Simplified energy diagram with the most important transitions in an Er:YAG $\left(50 \%\right.$ at $\left.\mathrm{Er}^{3+}\right)$ laser crystal
The laser operates at room temperature, and the laser emission that we are interested in develops between the states $I_{11 / 2}$ and $I_{13 / 2}$ at a wavelength of $2940 \mathrm{~nm} . \mathrm{I}_{11 / 2}$ is the laser upper level, while $\mathrm{I}_{13 / 2}$ is the laser lower level. The excitation with the Xe flash lamp directly pumps the levels ${ }^{4} \mathrm{~S}_{3 / 2}$ with a pumping rate $r_{l}\left(\mathrm{~cm}^{-3} \mathrm{~s}^{-1}\right)$ as well as the upper laser level $\mathrm{I}_{11 / 2}$ with the rate $r_{2}\left(\mathrm{~cm}^{-3} \mathrm{~s}^{-1}\right)$. The highly lying levels ${ }^{4} \mathrm{~S}_{3 / 2}$ are very quickly depleted with transitions to the upper laser level and therefore contribute to the creation of an inverse population. The up-conversion $w_{2}$ from the upper laser level pumps the ${ }^{4} \mathrm{~S}_{3 / 2}$ level. The up-conversion $w_{1}$ depletes the lower laser level in such a manner that it contributes to the inverse population twice. Both, the upper and lower laser levels are Stark split. The population distribution in the Stark split levels follows the Boltzmann distribution - i.e., the populations are determined by Boltzmann factors [11]. It is important to emphasize that both pumping rates $r_{1}$ and $r_{2}$ are tied with $r=r_{1}+r_{2}$. In this article we assumed the relationship between the rates $r_{1}$ and $r_{2}$ to be $k$ $=r_{2} /\left(r_{1}+r_{2}\right)[12]$.

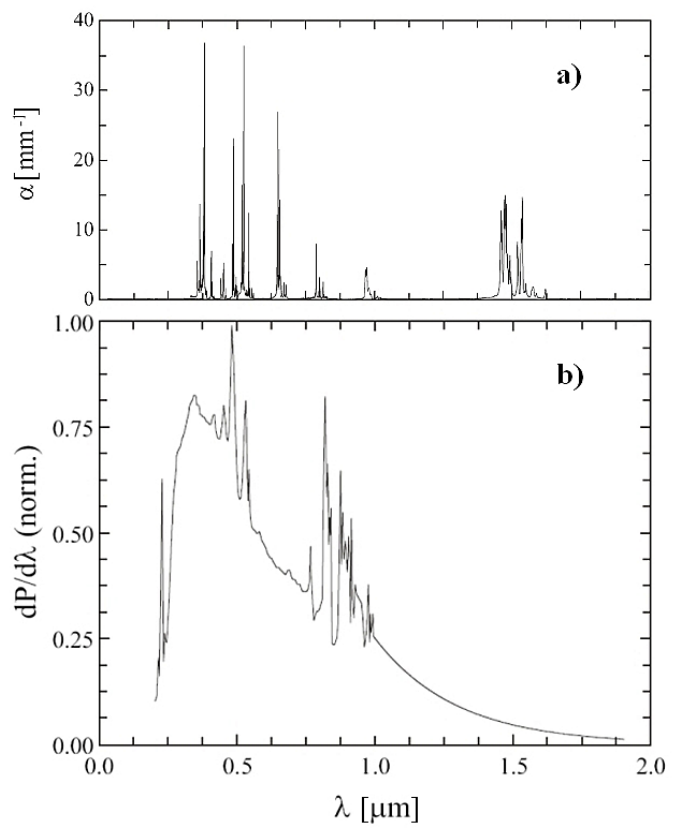

Fig. 3. a) 50\% doped Er:YAG crystal absorption spectrum; b) Xe flash lamp spectrum 
The Er:YAG laser is efficiently pumped by flash lamps filled with Xe since the pumping bands for the Er:YAG crystal are around 500, 650 and 750 to $800 \mathrm{~nm}$ and they overlap very well with the Xe flash lamp's spectrum (Fig. 3).

\section{POPULATION DYNAMICS OF AN Er:YAG LASER}

The time evolution of pumping, the population of various levels as well as stimulated emission is described with rate equations. Due to the many processes that are present during the Er:YAG laser pumping these equations are fairly complex. For studying the population dynamics of the upper and lower laser levels of a flashlamp-pumped Er:YAG laser as well as for the generation of laser emission, these equations can be simplified [12], and can be described using the equations below:

$$
\begin{gathered}
\frac{d n_{3}}{d t}=r_{1}(t)-\frac{n_{3}}{t_{3}}+w_{2} n_{2}^{2}, \\
\frac{d n_{2}}{d t}=r_{2}(t)+\frac{n_{3}}{t_{3}}+w_{1} n_{1}^{2}-2 \cdot w_{2} n_{2}^{2}- \\
-\frac{n_{2}}{t_{2}}-s_{21}\left(b_{2} n_{2}-a_{7} n_{1}\right) \Phi \\
\frac{d n_{1}}{d t}=\frac{n_{3}}{t_{3}}-2 \cdot w_{1} n_{1}^{2}+\frac{n_{2}}{t_{2}}-\frac{n_{1}}{t_{1}}+ \\
+s_{21}\left(b_{2} n_{2}-a_{7} n_{1}\right) \Phi \\
\frac{d \Phi}{d t}=s_{21}\left(b_{2} n_{2}-a_{7} n_{1}\right) \Phi-\frac{\Phi}{t_{c}},
\end{gathered}
$$

where is:

$n_{1} \quad$ lower laser level population $\left[\mathrm{cm}^{-3}\right]$

$n_{2} \quad$ upper laser level population $\left[\mathrm{cm}^{-3}\right]$

$n_{3} \quad$ population of ${ }^{4} \mathrm{~S}_{3 / 2}$ state $\left[\mathrm{cm}^{-3}\right]$

$\Phi \quad$ photon flux in the resonator $\left[\mathrm{cm}^{-2}\right]$

$r_{1}(\mathrm{t}) \quad$ pumping rate of ${ }^{4} \mathrm{~S}_{3 / 2}\left[\mathrm{~cm}^{-3} \mathrm{~s}^{-1}\right]$

$r_{2}(\mathrm{t}) \quad$ direct pumping rate of upper laser level $\left[\mathrm{cm}^{-3} \mathrm{~s}^{-1}\right]$

$s_{21} \quad$ cross-section for stimulated emission [ $\left.\mathrm{cm}^{2}\right]$

$b_{2} \quad$ Boltzmann factor of Stark spited $\mathrm{I}_{11 / 2}$ upper laser level

$a_{7} \quad$ Boltzmann factor of Stark spited $\mathrm{I}_{13 / 2}$ lower laser level

$t_{1} \quad$ lower laser level lifetime [s] $t_{2} \quad$ upper laser level lifetime [s]

$t_{3} \quad{ }^{4} \mathrm{~S}_{3 / 2}$ lifetime [s]

$w_{1} \quad$ coefficient of up-conversion process of upper laser level $\left[\mathrm{cm}^{3} \mathrm{~s}^{-1}\right]$

$w_{2} \quad$ coefficient of up-conversion of lower laser level $\left[\mathrm{cm}^{3} \mathrm{~s}^{-1}\right]$

$t_{\mathrm{c}} \quad$ photon lifetime in laser resonator [s].

The inversion population that is important for the laser emission is:

$$
n=\left(b_{2} n_{2}-a_{7} n_{1}\right),
$$

here, the degeneracy due to the Stark splitting of the upper and lower laser levels is taken into account.

The pumping rates' time behaviors $r_{l}(t)$ and $r_{2}(t)$ are used in these equations as $r_{1}(t)=$ $r_{1} P(t)$ and $r_{2}(t)=r_{2} P(t)$, where $P(t)$ is the shape of the flash-lamp pumping pulse with an amplitude of 1 . The relation between the pumping rates is a constant throughout, taken as $k=r_{2} /\left(r_{1}+r_{2}\right)=0.6$.

In order to check the above rate equations, they were numerically solved for a free-running Er:YAG laser (no Q-switching) for a rectangular flash-lamp current pulse of $300 \mu$ s duration, where for reasons of simplicity we used $P(t)=1-h\left(t-t_{p}\right)$, where $h(t)$ is a Heaviside step function and $t_{p}$ is the duration of the rectangular current pulse.

The constants used in Eqs. (1) to (4) were taken from reference [12]. The photon lifetimes were calculated for a resonator with plane/plane mirrors, an out coupling mirror transmitivity of $85 \%$ at $2940 \mathrm{~nm}$, a $\phi 4 \times 90 \mathrm{~mm}$ laser rod and an overall resonator length of $178 \mathrm{~mm}$.

Typical results of the solutions are shown in Fig. 4.

Since the laser in free-running mode operates in mixed-mode operation, without limiting apertures inside the resonator, except for the ones at the laser-rod ends, we can obtain the laser efficiency curve by determining the time integral of the photon flux (proportional to the laser pulse energy) as well as the pumping rate (proportional to the flash-lamp pulse energy) for various pumping rates.

The curve in Fig. 5 predicts a linear relationship between the laser output energy on the flash-lamp pulse energy that is a typical result, obtained in all experiments for an Er:YAG laser operating in the free-running mode. Therefore, the 

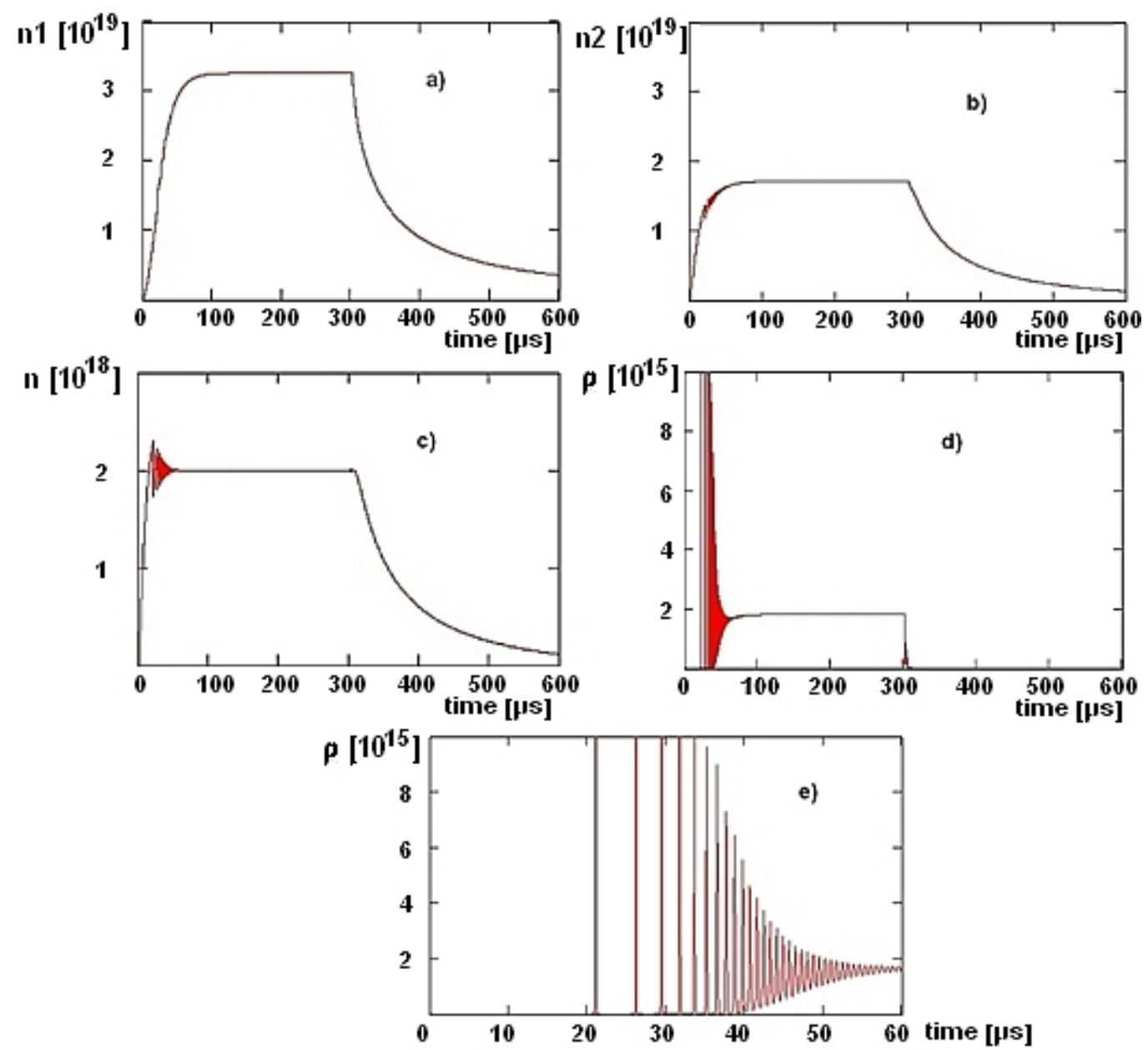

Fig 4. a) lower laser level population - n1; b) upper laser level population - n2; c) inverse population $\left.n=\left(b_{2} n_{2}-a_{7} n_{1}\right) ; d\right)$ photon flux in resonator $\left.-\rho ; e\right)$ photon flux in resonator with higher temporal resolution (note the relaxation oscillation micro pulses with a time duration of the order of $1 \mu \mathrm{s}$, which are also observed experimentally)

rate-equation model describes the behavior of a free-running Er:YAG laser well.

The curve in Fig. 5 predicts a linear relationship between the laser output energy on the flash-lamp pulse energy that is a typical result, obtained in all experiments for an Er:YAG laser operating in the free-running mode. Therefore, the rate-equation model describes the behavior of a free-running Er:YAG laser well.

\section{Q-SWITCHED OPERATION}

The simulation of the Q-switched operation was performed in two steps:

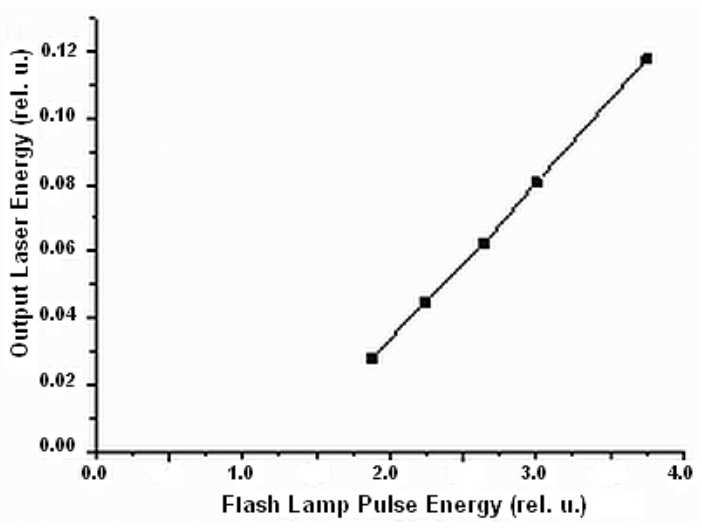

Fig 5. Er:YAG laser output energy as a function of the flash-lamp input energy 
a) From Eqs. (1) and (2), as well as from Eqs. (3) and (4), the inverse population as a function of time is determined, where we assume no laser action $(\Phi=0)$ is assumed. Here we assume a rectangular time profile of the flash-lamp current and hence the pumping terms $r_{l}(t)$ and $r_{2}(t)$. The inverse population is calculated until time equals $t_{0}$.

b) From time $t_{0}$ on, the rate Eqs. (1) to (3) and (6) are used, which were modified from Eq. (4) and presented below:

$$
\frac{d \Phi}{d t}=s_{21} \cdot n \cdot \frac{d_{2}}{l_{\text {res }}} \Phi-\frac{\Phi(L+\varepsilon(t))}{t_{R}} .
$$

Then, these equations, with the starting conditions from Eqs. (1) to (4) at time $t_{0}$, are solved numerically.

In Eq. 6 for a photon flux the term for the stimulated emission is multiplied by the ratio of the length of the active medium $d_{2}$ to the overall resonator length $l_{\text {res }}$. The photon lifetime $t_{c}$ is replaced with the resonator roundtrip time $t_{R}$ presented in Eq. 7:

$$
t_{R}=\frac{2 \cdot\left(d_{1}+n_{r o d} d_{2}+d_{3}\right)}{c},
$$

where $d_{1}$ is the separation between the out coupling mirror and the laser rod, $n_{\text {rod }}$ is the rod index of refraction, $d_{3}$ is the separation from the rod to the rear mirror center.

The losses in the resonator are expressed as:

$$
L=L_{s}+L_{o c},
$$

where $L_{s}$ are the diffraction losses and $L_{o c}$ are the losses from the out coupling mirror with scattering and absorption. $L_{s}$ was estimated to be $L_{s}=0.05$, while $L_{o c}$ was taken as:

$$
L_{o c}=-\ln \left(R_{o c}\right),
$$

where $R_{o c}$ is the reflectivity of the out coupling mirror.

The term $\varepsilon(t)$ is the time-dependent losses of the rotating-mirror Q-switch. $\varepsilon(t)$, as a function of the rotating mirror's angular frequency $\omega$, was modeled as [10]:

$$
\varepsilon(t)=a+b \cos (\omega t) .
$$

\section{ROTATING-MIRROR Q-SWITCHED LASER DESIGN}

The Q-switch in the form of a flat Si mirror (silver coated by II-VI Belgium) with a reflectivity of $99.7 \%$ at $2940 \mathrm{~nm}$ was fixed to a motor shaft. The position of the motor shaft at a defined angle $\alpha$ to the resonant position of the mirror was detected for triggering the flash-lamp discharge by optocouplers. The frequency of the rotating mirror was adjusted to $200 \mathrm{~Hz}$.

The Er:YAG laser-pumping chamber consisted of a $\mathrm{BaSO}_{4}$ diffuse reflector with a Borofloat 33 flow tube. A $4 \times 90 \mathrm{~mm}$ Er:YAG flat/flat rod (Sumitomo) doped with $50 \%$ at $\mathrm{Er}^{+3}$, AR coated at $2940 \mathrm{~nm}$ on both end faces, with fine ground sides, was used. The laser rod and the flash lamp were cooled with temperaturestabilized, de-ionized water $\left(T_{\text {water }}=18 \pm 0.5^{\circ} \mathrm{C}\right)$. The laser was pumped with a Xe flash lamp that had an inner diameter of $5 \mathrm{~mm}$ and an arc length of $89 \mathrm{~mm}$ with a cerium-doped envelope, made by Noblelight. The out coupling mirror was a flat/ flat mirror with a reflectivity of $85 \%$ at $2940 \mathrm{~nm}$ and an AR coating for $2940 \mathrm{~nm}$ manufactured by Layertec GmbH, Germany.

The laser resonator is shown in Fig. 6.

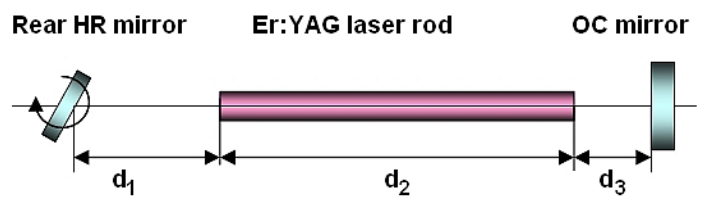

Fig. 6. Q-switched Er:YAG laser resonator, where $d_{1}=52 \mathrm{~mm}, d_{2}=90 \mathrm{~mm}$ and $d_{3}=36 \mathrm{~mm}$

The flash lamp was operated with a simmer current of approximately $150 \mathrm{~mA}$, while the main discharge (pumping pulse) was generated from a $10 \mathrm{mF}$ capacitor bank with an IGBT module. The IGBT module was activated with a programmable pulse generator, triggered by the rotating-mirror, Q-switch, position-sensor electronics. The repetition rate of the Q-switched laser pulses was set to $2.87 \mathrm{~Hz}$. The flash-lamp triggering circuit as well as the typical flash-lamp current and voltage pulses are shown in Fig. 7. During the experiments for each laser pulse, the flash-lamp current and 
the voltage pulse were measured and used for the calculation of energy delivered in the flash lamp.

\subsection{Simulations of Q-Switched Operation}

For reasons of simplicity, an ideal rectangular flash-lamp pumping pulse with a duration of $t_{p}=325 \mu$ s was used in the simulations. The real pumping pulse is not rectangular due to the time needed for the flash-lamp Xe plasma to fill the flash lamp. Since we were only interested in the creation of a certain inverse population, the shape of the pumping pulse did not influence the results of the simulation. The values of the populations of all three levels $n_{1}, n_{2}$ and $n_{3}$ were solved at the time $t_{0}$ with a photon flux $\Phi=0$. For times $t>t_{0}$ the photon flux was solved from Eqs. (1), (2), (3) and (6), where the time-dependent term for Q-switch losses $\varepsilon(t)$ was used. The simulations were made considering a Q-switch rotation frequency of $200 \mathrm{~Hz}$. See Fig. $8 \mathrm{a}$.
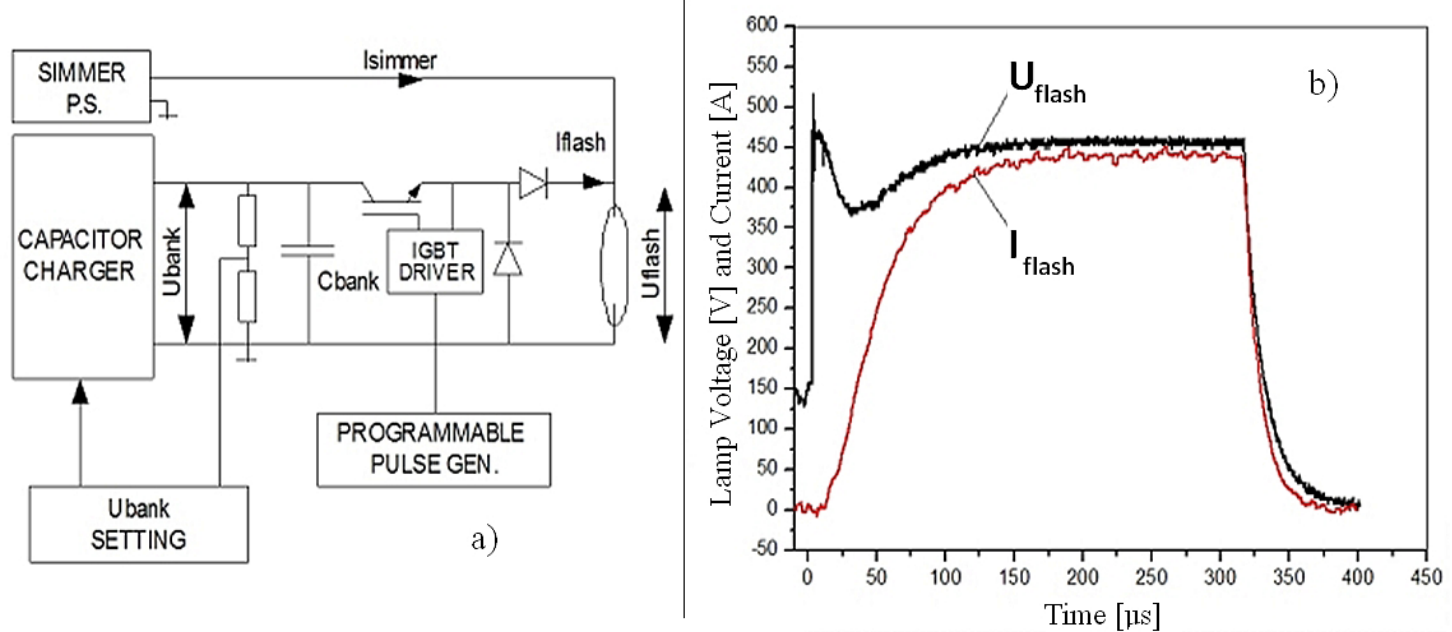

Fig. 7. a) flash-lamp triggering and main discharge control circuit and; b) typical flash-lamp voltage and current pulse shapes
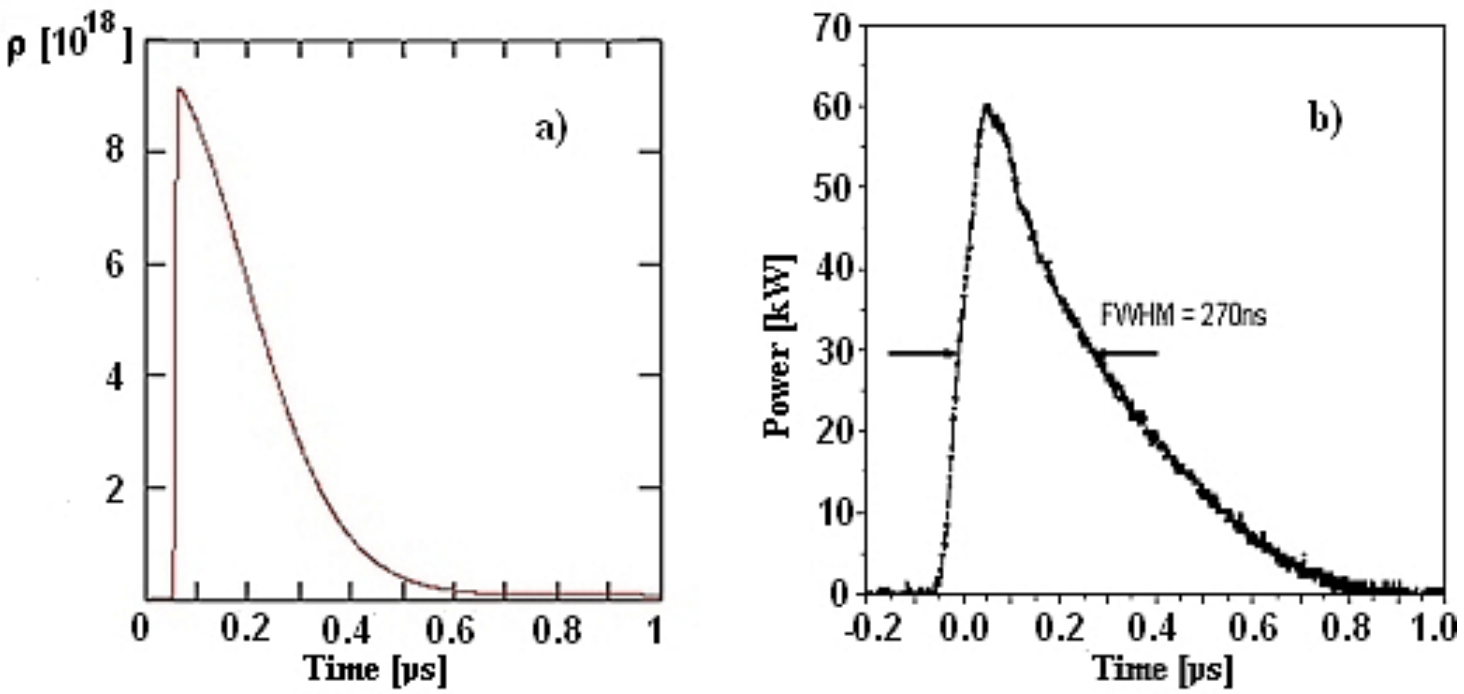

Fig. 8. Q-switched Er:YAG TEM 00 laser pulse temporal shape; a) calculated from rate Eqs. (1) to (3) and (9); b) experimentally realized and measured 


\subsection{Measured Properties of Q-Switched Er:YAG Laser}

The Q-switched laser pulse shape, energy, efficiency, output-energy repeatability and laserbeam spatial profile were measured. The measured Q-switched pulse temporal shape is shown in Fig. $8 \mathrm{~b}$, together with the same laser pulse calculated from the rate equations (Fig. 8a). The temporal pulse shape was measured with a fast InAs detector (Teledyne Judson Technologies).

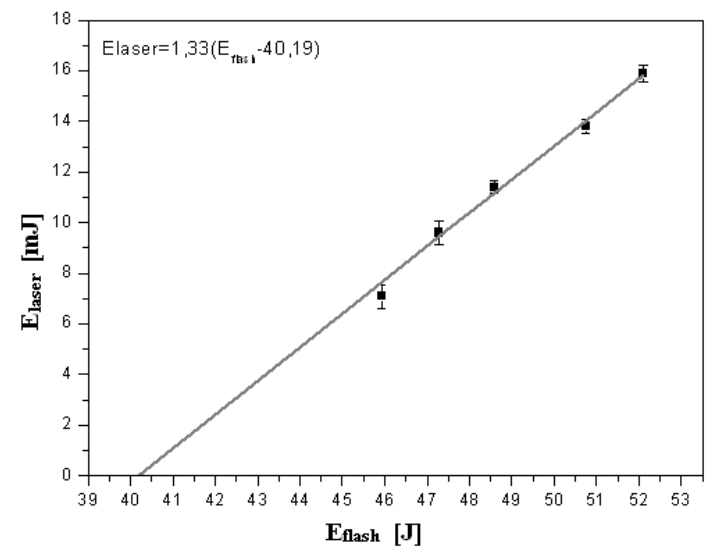

Fig. 9. Q-switched Er:YAG laser pulse output energy as a function of flash-lamp input energy; the threshold energy for $Q$-switched operation was found to be $40.2 \mathrm{~J}$

The Q-switched laser-pulse temporal shape remained the same for input energies in the flash lamp of $E_{\text {in }}=52 \mathrm{~J}$, with a pulse width (FWHM) of $270 \mathrm{~ns}$. At higher pumping energies, the evolution of the second pulse was observed (typical in rotating-mirror Q-switching [10]) as well as the evolution of higher transverse modes, since no mode selection in the form of additional limiting apertures was used in the laser design.

The laser output energy for single-mode, Q-switched operation as a function of the flashlamp input energy is shown in Fig. 9.

The repeatability of the laser pulses was measured at different laser-pulse energies (see Table 1) for a pulse-repetition frequency of 2.87 $\mathrm{Hz}$. The repeatability is the worst at low energies (approximately 7\%). For laser-pulse energies greater than $10 \mathrm{~mJ}$ the repeatability saturates to a remarkable value of $2 \%$.
Table 1. Calculated pulse-to-pulse repeatability

\begin{tabular}{|c|c|c|c|}
\hline $\begin{array}{c}\boldsymbol{E}_{\text {flash }} \\
{[\mathbf{J}]}\end{array}$ & $\begin{array}{c}\boldsymbol{E}_{\text {laser }} \\
{[\mathbf{m J}]}\end{array}$ & $\begin{array}{c}\text { St. dev. } \\
{[\mathbf{m J}]}\end{array}$ & $\begin{array}{c}\text { Pulse-to-pulse } \\
\text { repeatability [\%] }\end{array}$ \\
\hline $\mathbf{4 5 . 9}$ & 7.1 & 0.5 & $\mathbf{6 . 9}$ \\
\hline $\mathbf{4 7 . 3}$ & 9.6 & 0.4 & $\mathbf{4 . 6}$ \\
\hline $\mathbf{4 8 . 6}$ & 11.4 & 0.2 & $\mathbf{2 . 1}$ \\
\hline $\mathbf{5 0 . 8}$ & 13.8 & 0.3 & $\mathbf{2 . 1}$ \\
\hline $\mathbf{5 2 . 1}$ & $\mathbf{1 5 . 9}$ & $\mathbf{0 . 3}$ & $\mathbf{1 . 9}$ \\
\hline
\end{tabular}

The laser-beam spatial profile was measured at a position $341 \mathrm{~mm}$ from the laseroutput coupler. Due to a lack of equipment for better measurements, a technique of measuring the transmission through the apertures of various diameters was used and then the fluence as a function of aperture diameter and finally fitting the fluence to the Gaussian beam profile was calculated. The result is shown in Fig. 10.

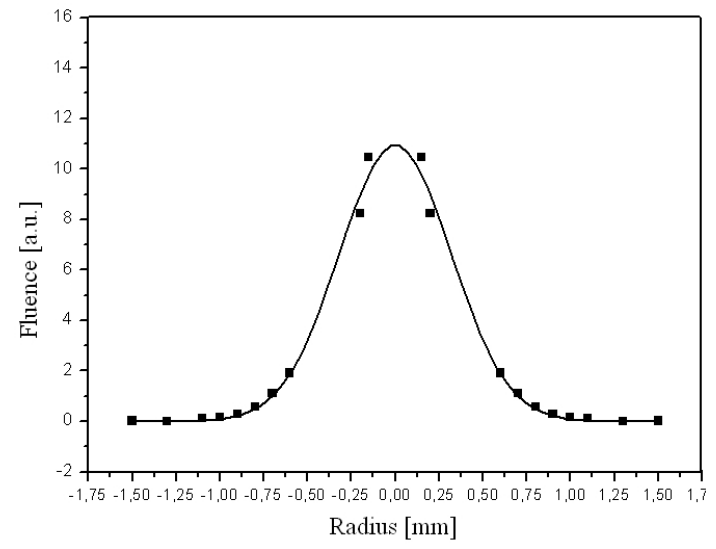

Fig. 10. Spatial profile of Er:YAG Q-switched laser beam at a distance of $341 \mathrm{~mm}$ from the laser out coupling mirror; the data points are from measured transmissions through apertures with various diameters; the laser-pulse energy was 15 $m J$ at repetition rate of $2.87 \mathrm{~Hz}$; the measured data points were fitted to a Gaussian beam profile (full line); the $1 / e^{-2}$ points are at $2 w=1.28 \mathrm{~mm}$

The measured spatial profile corresponds to a $\mathrm{TEM}_{00}$ mode with the $1 / \mathrm{e}^{-2}$ point at a radius of $0.64 \mathrm{~mm}$. A higher certainty could be reached with measurements made at multiple distances from the laser out coupling mirror.

The propagation of the fundamental laser mode created in the resonator was also calculated. 
Here, the thermal lensing of the laser rod under the conditions of the experiment (laser rod pumping power $=52.1 \mathrm{~J} \times 3 \mathrm{~Hz}=156.3 \mathrm{~W}$ ) was taken into account. The obtained result for the $1 / \mathrm{e}^{-2}$ point of the spatial profile was $0.85 \mathrm{~mm}$. For the reasons for the discrepancy between the measured and calculated value of the $1 / \mathrm{e}^{-2}$ point in the spatial $\mathrm{TEM}_{00}$ beam profile may lie in the inaccuracy of the method of the beam-profile measurement used. The other possible reason could be that in the calculation of the $\mathrm{TEM}_{00}$ mode and its propagation we took an average value of thermal lensing of the Er:YAG rod. The actual thermal lensing of the laser rod during the creation of the Q-switched laser pulse could be different than the average value we used.

\section{CONCLUSIONS}

We developed a simple, repeatable and reliable Q-switched Er:YAG laser for laboratory use. Q-switched, laser-pulse energies from 7 to $16 \mathrm{~mJ}$ with a pulse width of $270 \mathrm{~ns}$ and a $\mathrm{TEM}_{00}$ spatial beam profile were realized at a repetition rate of $2.87 \mathrm{~Hz}$. The pulse-to-pulse repeatability reached values of approximately $2 \%$.

The laser is intended for various optodynamic laboratory studies of $2940 \mathrm{~nm}$, Q-switched, laser pulses' interaction with various media that contain water. In the future, we intend to run experiments to detect and define the thickness of the water condensing on various surfaces, like aluminum, copper and glass.

\section{REFERENCES}

[1] Grad, L., Možina, J., Šušterčič, D., Funduk, N., Skalerič, U., Lukač, M., Cenčič, S., Nemeš, K. (1994). Optoacoustic studies of Er:YAG laser ablation in hard dental tissue. SPIE Proc., vol. 2128, p. 456-465.

[2] Jelínková, H., Dostálová, T., Hamal, K., Krejsa, O., Kubelka, J., Procházka, S. (1998). Er:YAG Laser in dentistry. Laser Physics, vol. 8, no. 1, p. 176-181.

[3] Paghdiwala, A.F., Vaidyanathan, T.K., Paghdiwala, M.F. (1993). Evaluation of erbium:YAG laser radiation of hard dental tissues: analysis of temperature changes, depth of cuts and structural effects. Scanning Microsc., vol. 7, no. 3, p.989-997.

[4] Shori, R.K., Walston, A.A., Stafsudd, O.M., Fried, D., Walsh, J.T., Jr. (2001). Quantification and modeling of the dynamic changes in the absorption coefficient of water at $\lambda=2.94 \mu \mathrm{m}$. IEEE Journal on Selected Topics in Quantum Electronics, vol. 7, no. 6, p. 959-970.

[5] Samokhin, A.A., Vovchenko, V.I., Ilichev, N.N. (2008). Photoacoustic and evaporation pressure signals in water irradiated with erbium laser pulses. Phys. Wave Phenomena, vol 16, no. 4, p. 275-282.

[6] Livingston, F.E., George, S.M., Shori, R.K. (2002). Optimization of rotary Q-switched Er:YAG laser. Rev. Sci. Instrum, vol. 73, no. 7, p. 2526-2532.

[7] Vodopyanov, K.L., Shori R.K., Stafsudd, O.M. (1998). Generation of Q-switched Er:YAG pulses using evanescent wave absorption in ethanol. Appl. Phys. Lett., vol. 72, no. 18, p. 2211-2213.

[8] Korada, P., Nemec, M., Jelinkova, H., Sulc, J., Cech, M., Shi, Yi-Wei, Matsuura, Y., Miyagi, M. (2005). Electro-optically Q-switched Er:YAG laser and its application. Proceedings of the SPIE, XV International Symposium of Gas Flow, Chemical Lasers, and High Power Lasers, vol. 5777, p. 384389.

[9] Lukač, M., Nemeš, K., Cenčič, S. (1993). Influence of direct and cross-relaxation pumping processes on the output energy and thermal load of an Er:YAG laser. Appl. Opt., vol. 32, p. 7399-7401.

[10] Koechner, W. (1999). Solid state laser engineering, $4^{\text {th }}$ ed. Springer-Verlag, Berlin.

[11] Pollack, S.A., Chang, D.B., Birnbaum, M., Kokta, M. (1991). Upconversion-pumped 2.8-2.9 $\mu \mathrm{m}$ lasing of $\mathrm{Er}^{3+}$ ion in garnets. $J$. Appl. Phys., vol. 70, p. 7227-7239.

[12] Majaron, B., Rupnik, T., Lukač, M. (1996). Temperature and gain dynamics in flash lamp-pumped Er:YAG. IEEE J. Quantum Electron, vol. 32, no. 9, p 1636-1644. 